\title{
Support Vector Machine Based on Incremental Learning for Malware Detection*
}

\author{
Zhuang Weiwei ${ }^{1}$ \\ ${ }^{1}$ School of Computer \& Information Engineering \\ Xiamen University of Technology \\ Xiamen, China \\ Zhuangweiwei@xmut.edu.cn
}

\author{
Xiao Lei ${ }^{2}$, Cui JianFeng ${ }^{1}$, Zhuang WeiChuan \\ ${ }^{2}$ Software Engineering Department of \\ Xiamen Institute of Software Technology \\ Xiamen, China \\ 1xiao@xmut.edu.cn
}

\begin{abstract}
The training of traditional SVM method requires the solution of quadratic programming, and consumes high memory and has low speed for large data training. Incremental learning is one of the meaningful methods to continuously update the data for learning, which keeps the previous learning results, re learning only for the additional data, so as to form a continuous learning process. This paper will study the support vector machine based on incremental learning method and its application in the malware detection. The experiments carried out in the Internet Security Laboratory at Kingsoft Corporation suggested that, for large number of virus samples, our method can rapidly and effectively update the sample features, which avoids duplication of learning history samples and ensures the malware prediction ability for the detection model.
\end{abstract}

Keywords-Support Vector Machine; Incremental Learning; Malware Detection

\section{INTRODUCTION}

With the development of Internet and software technology, computer virus renovates unceasingly, and its quantity growths sharply. Traditional scanning technology based on characteristic code faces a rigorous challenge ${ }^{[1]}$. In order to overcome the defects, data mining and machine learning methods, such as Naïve Bayes, Support Vector Machine and Decision Tree technology, are introduced to the field of antivirus $^{[2]}$. Paper ${ }^{[3]}$ use the windows API function sequence called by $\mathrm{PE}$ files as the feature to produce the association classification rules, which construct classifiers and defense against viruses. However, with the number of sample increase day by day, how to avoid re learning of historical data and perform incremental learning for the new sample to ensure the predictive ability of the classification model are still to be solved.

At present, there are few researches about incremental learning of the classification model. Cheung et al ${ }^{[4]}$ proposed the FUP algorithm, which use the frequent item sets obtained to updating association rules rapidly and reduces the update cost greatly. Paper ${ }^{[5,6]}$ present some incremental algorithms for updating association rules based on FP tree. Paper ${ }^{[7]}$ solves the problem of how to generate the frequent item sets in transaction database when the minimum support is unchanged. But these studies only maintain association rules generated by transaction database without the class attributes.

This paper presents a support vector machine method based on incremental learning called SVMIL (Support Vector Machine Based on Incremental Learning), the method can deal well with the class attribute data set, avoid repeating the study of before training samples, which based on the samples already learned to analysis and train new ones, and greatly improve the learning efficiency. The experimental results show that, for increasing training samples, our SVMIL method can rapidly, effectively maintain the sample features, and ensure the prediction ability of the detection model when applied to malware detection.

\section{RELATED WORK}

The traditional malware detection model based on classification method use the following procedure to detect virus ${ }^{[3]}$ :

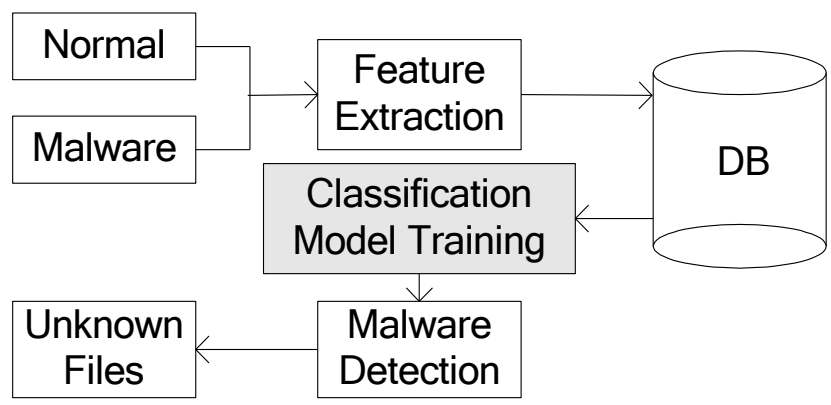

Fig. 1. The Traditional Malware Detection Model Based on Classification

The traditional malware detection model contains the following three modules:

(1) Feature extraction module: Collect features of the normal and the malware samples and store them in the database;

* Responding author, zhuangweiwei@xmut.edu.cn. Supported by the High-level Talents Project of Xiamen University of Technology under Grants No.YKJ13023R, the Research Fund of Xiamen University of Technology under Grants No.XYK201431, the Foreign Exchange and Cooperation Project of Xiamen University of Technology under Grants No.E201401500, and the Project of Xiamen Science and Technology Program under Grant No.3502Z20133043. 
(2) Training module: Build classification model by mathematics method based on the sample features;

(3) Malware detection module: Extract feature from unknown file, and determine whether it is a malware through the classification model constructed.

The classification model training is the key to the detection procedure. Apriori and FP-growth are two kinds of the methods commonly used for sample classification. The Apriori algorithm is time-consuming which needs to generate a huge number of candidate item sets in the mining process and needs for repeated scanning the entire database. FP-growth algorithm adopts the pattern growth method for mining association rule and overcomes the defect of Apriori. But it needs to recursively generate conditional FP-tree in mining frequent patterns, and each frequent pattern will generate a conditional FP-tree. When the threshold of the minimum support is lower, it may also generate a large number of frequent patterns for small database, which will consume a large amount of time and space.

The Fast_FP-growth algorithm ${ }^{[8]}$ introduced the concept of constraint subtree without generating conditional FP-tree. The efficiency of the algorithm is obviously higher than the traditional Apriori and the FP-growth. But without considering the efficient incremental updating of the association rules, the practical application of the algorithm is severely limited. Experiments show that, using Fast_FP-growth algorithm in large data set consumes a large amount of time and space, and is easy to cause the combinatorial explosion problem of the frequent item sets.

In the traditional model, the new samples and the history samples are put together then trained together. With the accumulation of samples, one-time mining problem which inevitably lead to the time and space overhead has become more and more serious. In order to solve the problem, this paper proposes an improved SVM classification method based on incremental learning. Different from the traditional ones, the method is able to handle well with the class attribute data set. By dividing the samples into small pieces and then incremental learning them one by one to update the classification model, the method will avoid repeated study and greatly improving the learning efficiency.

\section{THE SVMIL METHOD BASED ON INCREMENTAL LEARNING}

\section{A. The Improved Malware Detection Model}

Our proposed detection model is shown in figure 2. Different with the traditional detection model (Figure 1), the improved model retains the old support vectors and adds them to the new dataset for incremental training together, which avoids the low efficiency and time-space overhead problem with repeated learning of historical data.

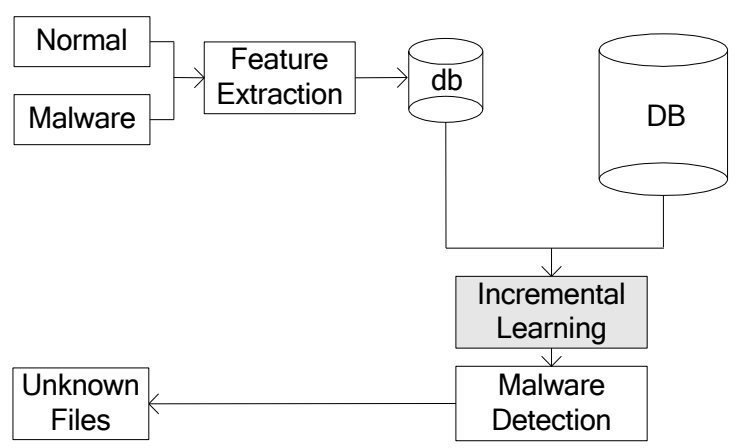

Fig. 2. The Improved Detection Model Based on Incremental Learning

\section{B. Basic Concepts and Definitions}

Given samples of $\mathrm{k}$ class, we construct an algorithm to distinguish each category. The purpose is to correctly classify the unknown samples. For the k class problem, the mathematical expression is as follows: Given k element sets $\mathrm{A}^{\mathrm{t}}$ in $\mathrm{n}$-dimensional space, where $\mathrm{t}=1,2, \ldots, \mathrm{k}$. Each element set contains $\mathrm{L}^{\mathrm{t}}$ elements $\mathrm{x}_{\mathrm{i}}^{\mathrm{t}}, \mathrm{i}=1,2, \ldots, \mathrm{L}^{\mathrm{t}}$, which are all belong to the same category. For each element set $\mathrm{A}^{\mathrm{t}}$, we looking for a ball $\left(\mathrm{c}^{\mathrm{t}}, \mathrm{r}^{\mathrm{t}}\right)$ that minimal enough to contain all (or almost all) of the sample points, where $c^{t}$ and $r^{t}$ are the center and the square of the radius of the ball. We allow outside points of the ball for the definition is very sensitive to some remote points. Similar to support vector machine, slack variable $\xi_{\mathrm{i}}{ }^{\mathrm{t}}$ is introduced here to get the following constraints:

$$
\begin{aligned}
& \left\|x_{i}^{t}-c^{t}\right\| \leq r^{t}+\xi_{i}^{t} \\
& \xi_{i}^{t} \geq 0 \quad i=1,2, \ldots, L^{t}
\end{aligned}
$$

The following object function can get the minimum slack variable value and the smallest square of the radius:

$$
\mathrm{F}\left(r^{t}, c^{t}, \xi_{i}^{t}\right)=r^{t}+S^{t} \sum_{i} \xi_{i}^{t}
$$

$\mathrm{S}^{\mathrm{t}}$ is a specified constant actually to control the punishment degree of the function for misclassified samples. Given a testing sample $\mathrm{x}$, we calculate the square of the distance from $\mathrm{x}$ to each center of the balls, that is :

$$
\left(x-c^{t}\right)^{2}=x^{2}-2\left(x * c^{t}\right)+\left(c^{t}\right)^{2}
$$

Then compare them with $\mathrm{r}^{\mathrm{t}}$,

$$
\left(c^{\mathrm{i}}\right)^{2}-2\left(\mathrm{x} * \mathrm{c}^{\mathrm{i}}\right)-\mathrm{r}^{\mathrm{i}}
$$

And find the category the minimum value belongs to which is the classification result of the sample.

Usually, even excluding remote samples, data are still not having a spherical distribution. To make the method presented in this paper applies more widely, and the sample feature can be transformed into a higher dimensional space, like SVM method, the inner product in the above formula can be replaced by the kernel function. The quadratic programming of the minimum ball is transformed into follows:

$$
\operatorname{maxL}\left(\alpha_{i}^{t}\right)=\sum_{i} \alpha_{i}^{t} k\left(x_{i}^{t}, x_{i}^{t}\right)-\sum_{i, j} \alpha_{i}^{t} \alpha_{j}^{t} k\left(x_{i}^{t}, x_{j}^{t}\right)
$$




$$
\text { s.t. }\left\{\begin{array}{l}
\sum_{i} \alpha_{i}^{t}=1 \\
0 \leq \alpha_{i}^{t} \leq S^{t}
\end{array}\right.
$$

And the formula for discrimination is:

$$
\mathrm{k}\left(c^{i}, c^{i}\right)-2 k\left(x, c^{i}\right)-r^{i}
$$

Different kernel function can lead to different feature spaces with different sample distribution shape, which are more convenient and accurate than the strict spherical distribution. In order to limit the transformation into higher feature space, we use the radial basis function kernel which is more suitable for the ball classification as follows:

$$
K(x, y)=\exp \left(\frac{-\|x-y\|^{2}}{2 \sigma^{2}}\right)
$$

\section{The SVMIL Algorithm}

In this paper, based on the ball classification method and the incremental learning, we retain the old support vectors and add them to the new data set for training as shown below:

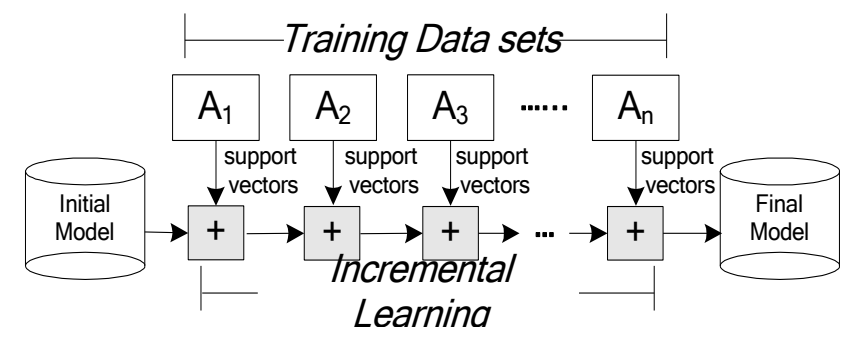

Figure 1. The Incremental Learning Procedure

The obtained support vectors in the ball classification are actually the points on the boundary of each category, which are more than the number of support vectors on the traditional SVM super plan. So this kind of incremental learning method can be well integrated with the ball classifier. The radius of the ball of each category is also decided by these support vectors. And the support vectors will not change in the category when the newly added data is surrounded by the

\begin{tabular}{|c|c|c|}
\hline \multicolumn{3}{|c|}{$\begin{array}{l}\text { Input : The first subset of data } A_{1} \text {, and the data with class label t in } A_{1} \\
\text { represent for } A_{1}{ }^{t} \\
\text { Output: The classification model }\end{array}$} \\
\hline 1 & Loop: & \\
\hline 2 & Input $A_{i}$ & // Input the ith training data set \\
\hline 3 & For each class $t$ in $A_{i}$, Do & \\
\hline 4 & $\begin{array}{l}\qquad \mathrm{SV}_{\mathrm{i}}^{\mathrm{t}}=\text { BallClassify }\left(\mathrm{A}_{\mathrm{i}}^{\mathrm{t}}\right) \\
\text { train the ith data set and obtain }\end{array}$ & $\begin{array}{l}\text { // Use ball classification method to } \\
\text { he support vectors }\end{array}$ \\
\hline 5 & $\mathrm{SV}=\sum_{t=1}^{k} S V_{i}^{t}$ & // Update the support vectors \\
\hline 6 & $\mathrm{~A}_{\mathrm{i}}^{\mathrm{t}}=\mathrm{A}_{\mathrm{i}}^{\mathrm{t}}+\mathrm{SV}^{\mathrm{t}}$ & \\
\hline 7 & $A_{i}=\cup A_{i}^{t}$ & $/ /$ Get the next batch of training set \\
\hline 8 & End For & \\
\hline 9 & Do Loop or Exit & til all the data sets have been trained \\
\hline
\end{tabular}
ball. Process of the SVMIL algorithm is as follows:

\section{TABLE I. SVMIL ALGORITHM}

Ball classification formula does not change in each training session, while only the center and the radius of each batch of data set will change with the change of the support vectors.

\section{EXPERIMENTAL RESUlts AND ANALYSIS}

In this section, detection performance of the SVMIL method is compared with other common traditional classification methods.

\section{A. Experimental Data}

This experiment used 10,000 viruses and 10,000 normal programs for training, 5,000 virus samples and 5,000 normal ones for testing. All the samples were provided by Kingsoft antivirus laboratory. Part of the data

\begin{tabular}{|c|c|c|c|c|}
\hline \& Id & FileName & FileSort & APISeq & IntVectorOfAPI \\
\hline 1 & 01BC5 ... & 1 & kernel32.dll,loadlibrarya... & $1,2,3,4,5,6,7 \ldots$ \\
\hline 2 & $066 \mathrm{ae} 3 \ldots$ & 1 & user32.dll,exitwindowse... & $1,2,6,9,10,1 \ldots$ \\
\hline 3 & ob57b9... & 1 & kernel32.dll,loadlibrarya... & $1,2,3,4,5,6,7 \ldots$ \\
\hline 4 & Ora71b... & 1 & user32.dll,opendesktop... & $1,2,6,9,10,1 \ldots$ \\
\hline 5 & $166426 \ldots$ & 1 & kernel32.dll,findfirstfilea;... & $1,2,6,37,42 \ldots$ \\
\hline 6 & $1920 \mathrm{~b} 2 \ldots$ & 1 & user32.dll,exitwindowse... & $1,2,6,9,10,1 \ldots$ \\
\hline 7 & $04 F 4 B 4 \ldots$ & 1 & user32.dll,opendesktop... & $1,2,6,9,10,1 \ldots$ \\
\hline 8 & $07 A 106 \ldots$ & 1 & user32.dll,opendesktop... & $1,2,6,9,10,1 \ldots$ \\
\hline 9 & $09 F 0 F 6 \ldots$ & 1 & user32.dll,opendesktop... & $1,2,6,9,10,1 \ldots$ \\
\hline 10 & $107527 \ldots$ & 1 & user32.dll,opendesktop... & $1,2,6,9,10,1 \ldots$ \\
\hline
\end{tabular}
set is listed in the following table:

\section{TABLE II. PART OF The EXPERIMENTAL DATA SET}

In the table, "FileName" is the MD5 value of the sample file. "FileSort" represents the type of the PE file ( 0 for normal, and the remaining for virus). "APISeq" is the API name sequence called by the sample, and "IntVectorOfApi" for the sequence of the API corresponding number.

\section{B. Development Environment}

The experiment was carried out under the following environment:

- Pentium 4 CPU, $3.00 \mathrm{GHz}$

-2.00GB memory

- Windows XP system

- VS2010 IDE

- MySQL5.0 database

\section{Results and Analysis}

In order to use the SVMIL method for incremental learning, the 20,000 samples are divided into 9 parts: $\mathrm{D}=\{\mathrm{D} 0, \mathrm{D} 1, \mathrm{D} 2, \ldots, \mathrm{D} 8\}$. D0 contains 4,000 samples, half normal and half malware. The rest divisions each contain 2,000 samples. D1 D4 are virus files, while D5 D9 are the normal ones. Taking D0 as the initial data set, we first train D0 to get the initial classification model, and then train the rest 8 sample sets one by one through the incremental learning method.

In order to compare the performance of SVMIL with other traditional classifier, we use the 20,000 samples described in 
this section as the training set and the other 10,000 samples as the test set. The experimental results are as follows:

TABLE III. ClassifiCATION PERFORMANCE OF DIFFERENT ClasSIFIERS

\begin{tabular}{|c|c|c|c|c|c|c|}
\hline Classifiers & TP & TN & FP & FN & Recall & Precision \\
\hline Decision Tree & 4093 & 4522 & 478 & 907 & $81.86 \%$ & $89.54 \%$ \\
\hline Naïve Bayes & 4130 & 4503 & 497 & 870 & $82.60 \%$ & $89.26 \%$ \\
\hline Fast_FPgrowth & 4421 & 4759 & 241 & 579 & $88.42 \%$ & $94.83 \%$ \\
\hline SVMIL & 4685 & 4854 & 146 & 315 & $93.70 \%$ & $96.98 \%$ \\
\hline
\end{tabular}

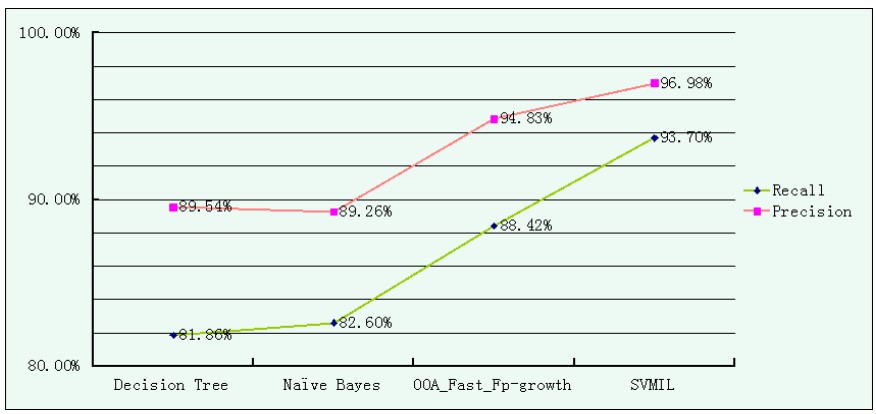

The predict results are evaluated by the precision rate and the recall rate which are defined as follows:

$$
\begin{aligned}
& \text { precision }=\frac{T P}{T P+F P} \\
& \text { recall }=\frac{T P}{T P+F N}
\end{aligned}
$$

Where TP(True Positive) is the correct classification number of the malware samples and TN(True Negtive) is the correct classification number of the normal ones, while FP(False Positive) represent the incorrect classification number of the malware and FN(False Negtive) represent the incorrect classification number of the normal files.

We can see from the experimental results, the decision tree algorithm is limited by the memory size due to the need for depth first search. In order to deal with large data set, all sorts of improvement (like discrete and sampling) not only increase the overhead of the algorithm, but also reduce the accuracy of classification. Completely fitting the training data has poor predict performance in the presence of noise. The premise of Bayes classification algorithm is the independence assumption, namely all attributes are independent to each other. But this assumption does not hold in malware classification, so its classification accuracy is not high. While using Fast_Fpgrowth algorithm, the lower the minimum support value is set, the higher confidence rules are likely to be get. But when the minimum support value is set to be 0.05 , the frequent item sets combination explosion problem will take up a lot of system resources, and the $2 \mathrm{G}$ memory is not enough for the algorithm. In this case, the detection accuracy and the coverage are all limited. The SVMIL algorithm reduces the scale of training samples, avoids repeated study, solves the one-time training problem for large data set under space-time limited, and improves the learning efficiency. In addition, the method takes full account of the relationship between the attributes of the samples and categories, which ensures the prediction capability of the trained classifier. Compared with other commonly used methods, SVMIL has better performance, highest detection rate and accuracy in the malware classification.

\section{CONCLUSIONS}

In this paper, we propose an improved method called SVMIL which is suitable for malware detection. Different from the traditional methods, it has the following advantages. First of all, the method can deal well with the class attribute data set. In the next place, the method divides the samples into pieces and updates the classification model continuously by incremental learning method, which can reduce the sample size for each study and effectively avoid the overhead problem brought about by the one-time mining of large data set. Finally, the classification does not interfere with the previous when adding a new class attribute to the data The previous calculation will still be effective, and all need to be done is apply a quadratic programming and simple calculation to the new classification itself, which makes the classification operation easier, no duplication of work, with more expansion ability, and is suitable for incremental data set. The experimental results show that, the SVMIL method is better than the traditional classification methods for less space-time resource consumption, which can rapidly updating the sample features, ensure the prediction ability of classification model, and can be effectively used for malware detection.

\section{ACKNOWLEDGMENT}

The authors would also like to thank the members of the Internet Security Laboratory at Kingsoft Corporation for their helpful discussions and suggestions.

\section{REFERENCES}

[1] Shui Yu, Guofei Gu, Barnawi, Song Guo, Stojmenovic. Malware Propagation in Large-Scale Network. Knowledge and Data Engineering, IEEE Transactions on, 2014, 27(1): 170-179.

[2] Acar Tamersoy, Kevin Roundy, Duen Horng Chau. Guilt by Association: Large Scale Malware Detection by Mining File-relation Graphs. In: Proceedings of KDD'14, August 24-27, 2014, New York, NY, USA, pages: 1524-1533.

[3] Yanfang Ye, Dingding Wang, Tao Li, and Dongyi Ye. IMDS: Intelligent malware detection system. In Proccedings of ACM International Conference on Knowlege Discovery and Data Mining (SIGKDD), 2007, page: 1043-1047.

[4] Cheung D.W. et al. Maintenance of discovered association rules in large databases: an incremental updating technique. In : Proceedings of the 1996 International Conference on Data Engineering , New Orleans , Louisiana , 1996 , page: 106-114

[5] Zhu Yu-Quan, Sun Zhi-Hui, Ji Xiao-Jun. Incremental updating algorithm based on frequent pattern tree for mining association rules. Journal of Computers , 2003 , 26 (1) : 91-96

[6] Yang Ming et al. Fast incremental updating of frequent itemsets. Journal of Applied Sciences, 2003, 21 (4): 367-372.

[7] Zhu Yu-Quan et al. Fast updating frequent itemsets. Journal of Computer Research and Development, 2003, 40(1): 94-99.

[8] Fan Ming, Li Chuan. Mining frequent patterns in an FP-tree without generating conditional FP-tree Generation [J]. Jouranl of computer research and development, 2003, 8(40): 1216-1222. 